\title{
Transepidermal elimination of lepromatous granuloma: a mechanism for mass transport of viable bacilli
}

\author{
M. NAMISATO*†, M. KAKUTA*†, K. KAWATSU \\ A. OBARA, S. IZUMI $\&$ H. OGAWA \\ * National Hospital Tama-Zenshoen, Aoba-cho 4-1-1, \\ Higashimurayama-shi, Tokyo 189, Japan; \\ $\dagger$ Department of Dermatology, Juntendo University School of \\ Medicine, Hongo 2-1-1, Bunkyo-ku, Tokyo, Japan; \\ $\ddagger$ National Institute for Leprosy Research, Tokyo, Japan; \\ $\S$ Department of Dermatology, Kyoto University Hospital, Kyoto, \\ Japan
}

Accepted for publication 7 October 1996

\begin{abstract}
Summary A 35-year-old male with lepromatous leprosy showed significant progression of the disease on initial examination. Along with typical lepromatous skin lesions, many scar-forming lesions were present, mainly on his extremities. Some lesions showed erosive surfaces. From clinicopathological findings, these lesions were suspected to be due to the partial excretion of intradermal lepromatous granulomata by 'transepidermal elimination'. Increased local volume, which might be due mainly to rapidly growing lepromatous infiltration before chemotherapy, is suspected of triggering this phenomenon. There is no doubt that many fresh Mycobacterium leprae were included in these excretions. After the initiation of chemotherapy, no new scar-forming lesions were observed.
\end{abstract}

\section{Introduction}

The details of transmission of leprosy are not clear, but the main portals of exit for Mycobacterium leprae (M. leprae) are generally assumed to be the skin and nasal mucosa. It is well known that a large quantity of $M$. leprae can be secreted from the nasal mucosa of patients with the lepromatous type of leprosy (LL). ${ }^{1}$ Although $M$. leprae are found in the epidermis and desquamating epithelium, ${ }^{2}$ the amount of these bacilli is considered to be very small from an infectious point of view. LL patients have a larger number of bacilli deep in the dermis; however, without skin ulcers or breakdowns, it might be difficult to detect these bacilli in large quantity on skin surfaces. ${ }^{3}$ We recently encountered scar-forming lesions on the limbs of an LL patient, and many fresh $M$. leprae were suspected of being excreted through these lesions. M. leprae can maintain prolonged viability under many different environmental conditions. ${ }^{4}$ From an epidemiological perspective, we should pay particular 
attention to skin lesions of leprosy patients, especially untreated active LL cases, and consider these lesions as one of the possible sources of infection.

\section{Case report}

The patient was a 35-year-old male of Japanese origin who was born and raised in Paraguay until the age of 29. No leprosy was reported in his family history.

He consulted a local hospital with many asymptomatic skin lesions that had persisted for about 1 year. On this initial examination, numerous pea-sized intradermal nodules, coexisting with slightly red papules, were noticed mainly on his extremities and buttocks. At the same time, disseminated scar-forming lesions were also noticed. Some of these had erosive surfaces. The biopsy specimens of the intradermal nodule and scar-forming lesion showed many rod-shaped bacilli on Fite's staining. Soon after the diagnosis of leprosy was established, $200 \mathrm{mg} /$ day of minocycline was administered. Thereafter, the patient was referred and admitted to the National Hospital Tama-Zenshoen. When we first examined his condition, 2 weeks after the initial examination, he had massively infiltrated earlobes and swollen digits as well as many nodules and papules, but no sign of madarosis. Skin smears from these areas showed that the BI was 5 3+ and the MI was 10 5\%. On his trunk, diffuse obscure erythematous lesions were noted with a $\mathrm{BI}$ of $2+\sim 0$. Histological findings of the papules and intradermal nodules disclosed typical lepromata. Based on all these findings, the patient was diagnosed with actively progressing LL.

Scar-forming lesions measuring $8 \sim 15 \mathrm{~mm}$ in diameter were seen mainly on the lateral aspects of the extremities, especially the distal extremities to the elbows or knees. Most lesions were composed of slightly red circular papules with atrophic hypopigmented central

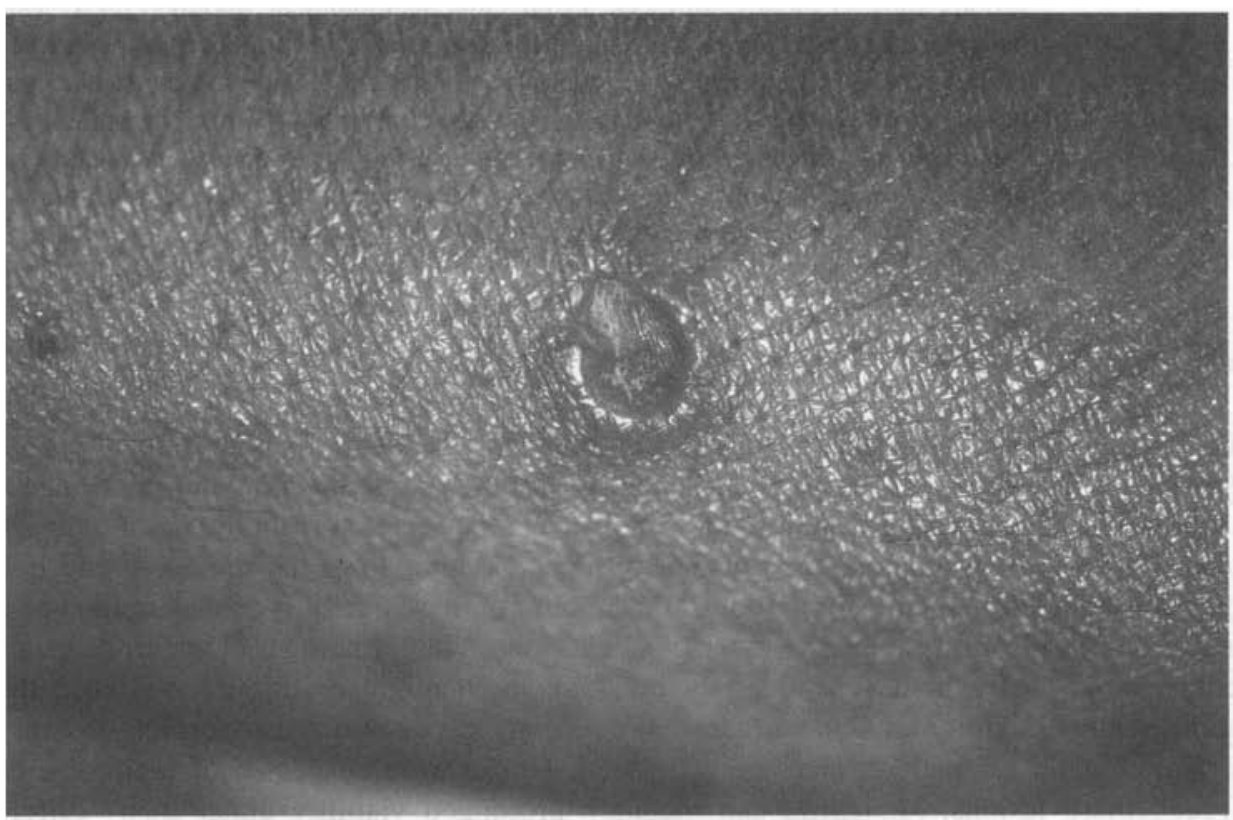

Figure 1. One of the umbilicated, scar-forming lesions on the lower leg. 
portions causing umbilication (Figure 1). Some of these had small crusts on the surfaces, but there was no further erosion. About 1 year earlier, the patient had noticed numerous small masses under the skin surface where scars later developed. He said they had gradually moved superficially followed by ulceration of the central portion from which pus-like material discharged. He had experienced no symptoms, and did not find any purpura or bulla-like lesions on his extremities during the whole course of developing these lesions. He also added that he had scratched some of these lesions.

After he was admitted to our hospital, WHO-multidrug therapy (WHO-MDT) ${ }^{5}$ for multibacillary leprosy was administered. Two months after the start of chemotherapy, he developed an apparent Type I leprosy reaction (reversal reaction), but it was well controlled by $30 \mathrm{mg} /$ day of prednisolone.

The following month, some of the scars on his extremities were excised for cosmetic reasons. During a one-year follow-up period, no new scar-forming lesion presented, and the central area of each umbilication has become shallower. While continuing MDT, he has returned to society and is currently employed.

\section{Histopathological findings of scar-forming lesions}

The biopsy specimen taken at the initial examination and the samples taken 3 months after chemotherapy was started are compared. The observation of longitudinal sections of all these specimens showed one or two residual hair follicles at the slightly protruded marginal areas.

Figure 2 shows the histology of the specimen taken at the initial examination, including

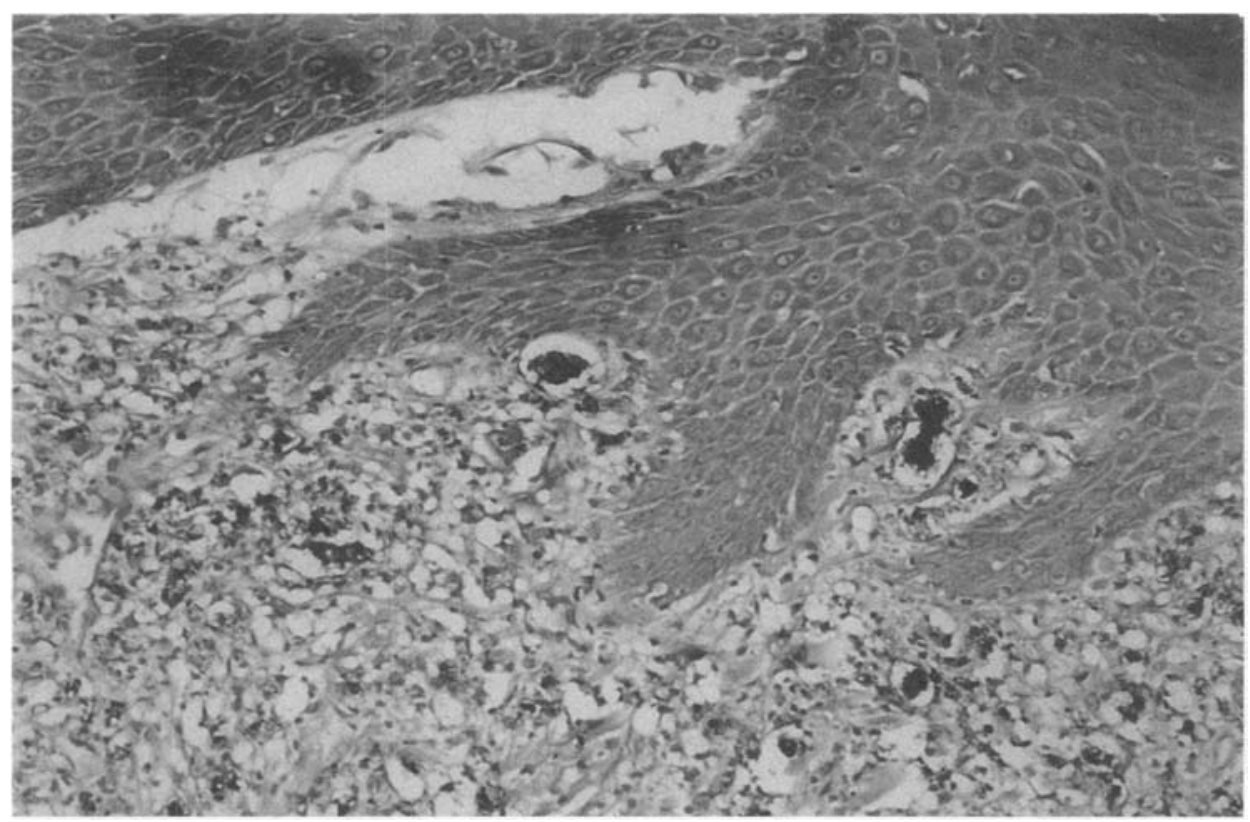

Figure 2. The histology of scar-forming lesion taken at the initial examination shows irregular acanthosis and invading tendency of the epithelium into the intradermal lepromatous granuloma which contains many rod-shaped acid-fast bacilli. (Fite's stain $\times 100$.) 
the marginal area (Fite's stain). The follicular and interfollicular epithelium showed irregular acanthosis and tended to invade the dermis in an effort to engulf the intradermal granuloma. Small parts of the granuloma were observed within the hyperplastic epithelium. The dermis was occupied by massive granuloma which consisted of characteristic foamy cells with many acid-fast bacilli (AFB). Most of these AFB were rod-shaped and some formed globi.

Figure 3 shows the histology of one of these lesions taken 3 months after the start of chemotherapy (hematoxylin-eosin stain). The structure of the hair follicle was apparent on each side of this specimen. Neither the epidermal hypertrophy nor the epidermal invasion into the dermis was seen anymore. Around the hair follicle, there was dense lymphocytic infiltration mixed with wasted (devitalized) leprous granuloma in which many granulated AFB were recognized by Fite's stain (not shown). At the flat central area of this specimen, the epidermis is atrophic, and the corium consists mainly of fibrous connective tissue.

\section{Discussion}

From the histopathological findings, some parts of intradermal lepromatous granuloma are suspected to have been expelled through the epidermis, and the follicular epithelium is thought to be strongly involved in this process. As a result, many fresh AFB must have been discharged.

Mehregan described the mechanism of eliminating intradermal materials in discussing the pathogenesis of elastosis perforans serpiginosa. ${ }^{6}$ According to his description, the

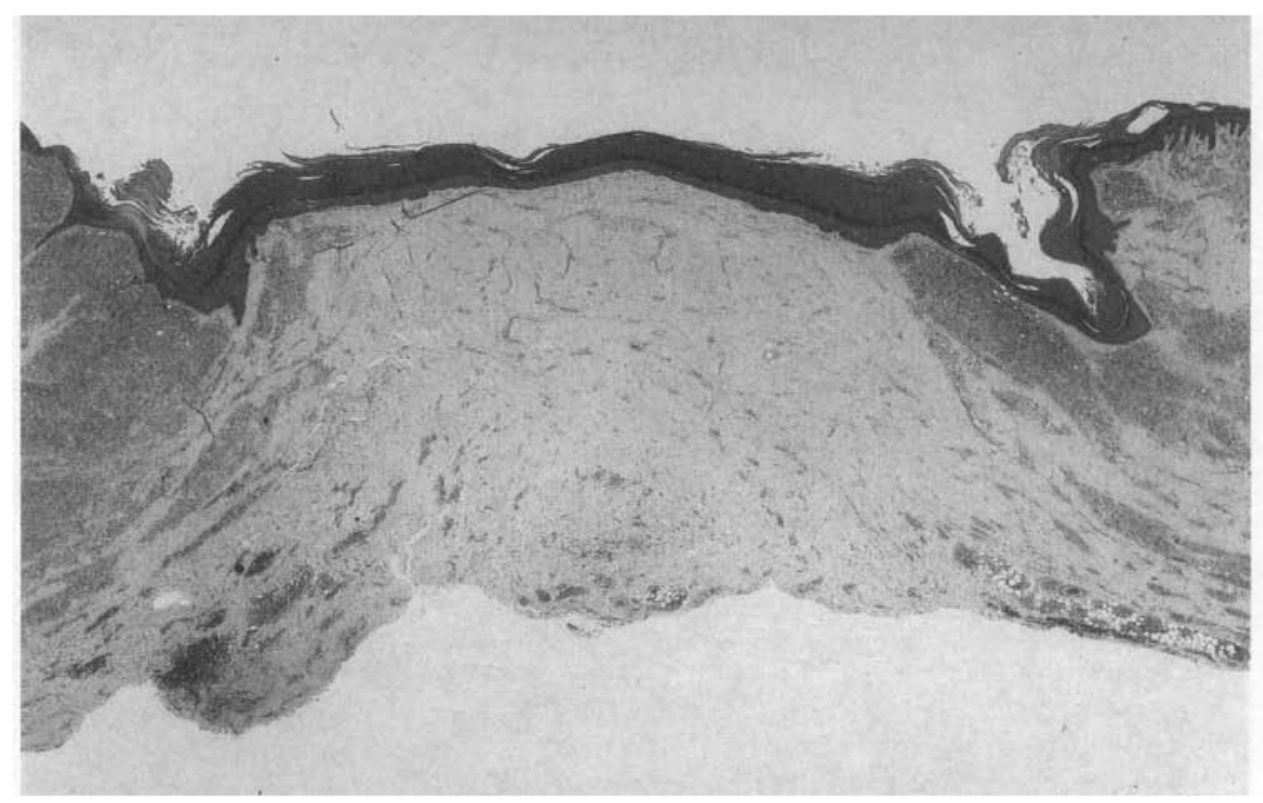

Figure 3. The histology of one of the scars taken at 3 months after chemotherapy shows a structure of hair follicle on each side of this specimen. Around the hair follicle, there is dense lymphocytic infiltration mixed with wasted leprous granuloma. (Hematoxylin-eosin stain $\times 10$.) 
phenomenon of 'transepidermal elimination (TEE)' seems to occur with non-irritating material. If the material to be eliminated is small, there is no significant derangement of the epithelium. On the other hand, if the material is composed of larger particles, varying degrees of pseudoepitheliomatous hyperplasia of the epidermis or follicular epithelium may occur in areas of contact with this material. Subsequently the hyperplastic epidermis may engulf the dermal material and eventual elimination may occur. Mehregan et al. added reactive perforating collagenosis (RPC) as another model of this phenomenon. ${ }^{7}$ In our case, umbilicated clinical features and histologically apparent hypertrophic changes of follicular epithelium engulfing the intradermal granuloma were very similar to that of RPC. To our knowledge, there has been no report of TEE of lepromatous granuloma involving engulfment by hypertrophic follicular epithelium.

The granuloma of LL is unique in that it can stay for a long time without receiving any rejection from the host. Normally, thin fibrous stroma called free zone intervenes between intradermal lepromatous granuloma and epidermis, but hair follicles are tightly surrounded by the granuloma without free zone. In the case discussed, the scars were located mainly on the anterolateral aspect of the extremities and these areas contain many terminal hairs especially in male.

Bayoumi ${ }^{8}$ observed the phenomenon of TEE using animal models and proved that follicular epithelium was more actively involved in this process. This case suggests that the terminal hair follicles which survived against invading lepromatous infiltration played a role in this phenomenon.

On his first visit, the patient's condition was highly progressive as shown by many rodshaped AFB. So the lepromatous infiltration might have been rapidly increasing in size until chemotherapy was started. Meanwhile, it is well known that LL cases can manifest Type II leprosy reaction even before the commencement of chemotherapy. One of the characteristics of leprosy reactions, including Type II reaction, is acute oedematous infiltration which becomes apparent in each local lesion. Rapidly increasing local volume caused by these conditions may stimulate follicular epithelium and trigger subsequent phenomenon.

Based on the fact that we could not find any erosive lesion when we first examined the patient, two weeks of chemotherapy seemed to be able to suppress this phenomenon. After MDT was started, even during the period of reversal reaction, there was no erosion of these lesions.

We should also discuss the superficial trauma which is most likely to precipitate skin lesions of RPC. ${ }^{7}$ Trivial traumas resulting from scratching the lesions might have contributed to this phenomenon to some extent.

Malak \& Kurban ${ }^{9}$ summarized the excretory function of the epidermis proposing the term 'catharsis'. They postulated that this mechanism could be observed in a variety of skin lesions and the close dermoepidermal interactions should have an important role in this mechanism. As one of the dermal factors which can influence this mechanism, they mentioned macrophages. Recently, there was a report about cutaneous leishmaniasis, ${ }^{10}$ which showed transepidermal elimination of causative parasites (Leishmania amastigotes) in large quantity.

It is interesting that both of cutaneous leishmaniasis and leprosy are considered to be deeply related to the dysfunction of dermal macrophages.

\section{Acknowledgment}

We are grateful to Dr Y. Fujita of Yamato City Hospital for his significant contribution to the preparation of pathological specimens for this case. 


\section{References}

1 Pedley JC. The nasal mucus in leprosy. Lepr Rev, 1973; 44: 33-35.

2 Okada S, Kimura J, Nishiura M. Mycobacterium leprae found in epidermal cells by electron microscopy. Int $J$ Lepr, 1978; 46: 30-34.

3 Pedley JC. Composit skin contact smears: a method of demonstrating non-emergence of Mycobacterium leprae from intact lepromatous skin. Lepr Rev, 1970; 41: 31-43.

4 Desikan KV, Sreevatsa. Extended studies on the viability of M.L. outside the human body. Lepr Rev, 1995; 66: 287-295.

5 WHO. Chemotherapy of leprosy for control programmes. WHO Technical Report Series No. 675, 1982.

6 Mehregan AH. Elastosis perforans serpiginosa. Arch Derm, 1968; 97: 381-393.

7 Mehregan AH, Schwartz OD, Livingood CS. Reactive perforating collagenosis. Arch Derm, 1967; 96: $277-282$.

8 Bayoumi AHM, Gaskell S, Marks R. Development of a model for transepidermal elimination. Brit J Derm, 1978; 99: 611-620.

9 Malak JA, Kurban AK. 'Catharsis': an excretory function of the epidermis. Brit J Derm, 1971; 84: 516-522.

10 Azadeh B, Abdulla F. Transepidermal elimination in cutaneous leishmaniasis. Acta Derm Venereol (Stockh), 1994; 75: 159 . 